VOL. $21(1980), 21-28$.

\title{
TRIVIALIZING RIBBON LINKS BY KIRBY MOVES
}

\author{
JONATHAN A. HiLLMAN
}

In this note it is shown that any ribbon link is a sublink of a ribbon link for which surgery on the longitudes gives a connected sum of copies of $s^{1} \times s^{2}$. In particular there are many links for which the analogue of the knot theoretic Property $R$ fails, and sublinks of homology boundary links need not be homology boundary links. Higher dimensional analogues of these results are also given and it is shown that if $n \geq 2$ the group of a $\mu$-component ribbon $n$-link has a presentation of deficiency $\mu$. Hence there are high dimensional slice knots which are not ribbon knots.

DEFINITION. A $\mu$-component $n$-link is a locally flat embedding $L: \mu S^{n} \rightarrow S^{n+2}$. It is a ribbon link if it extends to an immersion $R: \mu D^{n+1} \rightarrow S^{n+2}$ with no triple points and such that the components of the singular set are $n$-discs whose boundary $(n-1)$-spheres either lie on $\mu S^{n}=\partial\left(\mu D^{n+1}\right)$ ("throughcut") or are disjoint from $\mu S^{n}$ ("slit").

It is well known and easy to see that ribbon links are null concordant [3]. The converse remains an open conjecture when $n=1$, even for knots $(\mu=1)$ [3], but is false in higher dimensions [8], [19], as will be shown below.

DEFINITION. A $\mu$-component $n$-link $L$ is an homology boundary link if there an epimorphism $\pi: G(L) \rightarrow F(\mu)$, where $G(L)=\pi_{1}\left(s^{n+2}-i m L\right)$ is

Received 28 June 1979. 
the link group and $F(\mu)$ is the free group of rank $\mu$. It is a boundary link if it extends to an embedding of $\mu$ disjoint orientable $(n+1)-$ manifolds, each with one boundary component.

Smythe had conjectured that if the first Alexander ideal of a 2-component l-link were zero, then the link would have to be an homology boundary link [15]. In [6] I gave an example of a 2-component ribbon l-link which was not an homology boundary link, thus refuting this conjecture (for the first $\mu-1$ Alexander ideals of a null concordant $\mu$-component link must vanish [7]). This example and the following theorem show that a sublink of an homology boundary link need not be an homology boundary link, although a sublink of a boundary link is clearly a boundary link.

THEOREM 1. Let $L$ be a $\mu$-component ribbon n-link. Then $L$ is a sublink of a v-component ribbon n-iink $\hat{L}$ for which surgery on the zongitudes gives $\# S^{1} \times S^{n+1}$. In particular $\hat{L}$ is an homology boundary iink.

Proof. Let $R: \mu D^{n+1}+S^{n+2}$ be a ribbon extending $L$. Let $S_{i}$, $1 \leq i \leq \sigma$, be the slits of $R$ and for each slit choose a regular neighbourhood $N_{i}$ contained in the interior of the corresponding disc and such that $N_{i} \cap N_{j}=\emptyset$ for $i \neq j$. Let $\nu=\mu+\sigma$ and let $\hat{L}=R \mid\left(\mu S^{1} \cup \underset{i=1}{\sigma} \partial N_{i}\right) \cdot$ Clearly $\hat{L}$ is a $\mu$-component ribbon $n$-link with $L$ as a sublink. If $n>I$ the normal bundle of $\hat{L}$ in $S^{n+2}$ has an essentially unique framing; if $n=1$ give each component of $\hat{L}$ the o-framing. Let $W(\hat{L})=D^{n+3} \cup \cup D^{n+1} \times D^{2}$ where $T: \cup S^{n} \times D^{2} \rightarrow S^{n+2}$ is an embedding of a regular neighbourhood of $\hat{L}=T \mid v S^{n} \times\{0\}$ determined by this framing. Then $\partial W(\hat{L})$ is the result of surgery on $S^{n+2}$ along the longitudes of $\hat{L}$.

Now by adding a pushoff of $\hat{L} \mid \partial N_{i}$ to the component of $L$ bounding the $(n+1)$-disc containing $N_{i}, \hat{L}$ may be replaced by a ribbon link with 
one less singularity; moreover if $n=1$ each component of the newlink still has the o-framing. Continuing thus $\hat{L}$ may be replaced by a ribbon link $\tilde{L}$ for which the only singularities are those corresponding to the components $\partial N_{i}$. Clearly these components may be slipped off the ends of the other components of the new ribbon and so $\tilde{L}$ is a trivial $v$-component link. Now adding pushoffs of link components to one another (a Kirby move of type $2[10])$ corresponds to sliding $(n+1)$-handles of $W(\hat{L})$ across one another, which leaves unchanged the topological type of $W(\hat{L})$ and hence of $\partial M(\hat{L})$. Thus $\partial W(\hat{L})$ is homeomorphic to $\partial W(\tilde{L})$, $\partial W(\tilde{L})=\# S^{1} \times S^{n+1}$ and $G(\hat{L})=\pi_{1}\left(S^{n+2}-i m \hat{L}\right)$ maps onto $\pi_{1}(\partial W(\hat{L})) \approx F(\nu)$

If $n=1$ the kernel of the map $G(\hat{L}) \rightarrow F(\nu)$ is necessarily $G(\hat{L})_{\omega}=\prod_{n \geq 1} G(\hat{L})_{n}$, the intersection of the terms of the lower central series for $G(L)$ [17], and is trivial if and only if $\hat{L}$ is trivial, in which case $L$ is also trivial. If $n>1$ the map $G(\hat{L}) \rightarrow F(\nu)$ is an isomorphism, but need not carry meridians to a generating set. (Poenaru gave the first examples of this phenomenon in [13].) This is the case if and only if $\hat{L}$ is a boundary link [4]. In the latter case, since moreover $\pi_{i}\left(s^{n+2}-i m \hat{L}\right) \approx \pi_{i}\left(s^{n+2}-i m \tilde{L}\right)=0$ for $2 \leq i<n$, so if $n>2, \hat{L}$ (and hence $L$ ) is trivial, by Gutiérrez' unlinking theorem [4]. The above theorem is not the best possible, in that fewer new components may suffice to trivialize $L$ thus. For instance, if $L$ is the square knot, it is a component of a 2-component homology boundary link with the above property. Recalling that a l-knot is said to have Property $R$ if surgery on a longitude of the knot does not give $s^{1} \times s^{2}$, and that is has been conjectured that all nontrivial l-knots have Property $\mathrm{R}$ ([11], Problem 1.16), this example shows that the most direct analogue of Property $R$ for links fails already for a 2-component 1-link. However I know of no example of a boundary 1-11nk for which 0-framed surgery gives a connected sum of copies of $S^{1} \times S^{2}$. If such a link exists, the subgroup $G_{\omega}$ of the link group must be perfect $\left(G_{\omega}=G_{\omega}^{\prime}\right)$ for the longitudes of a boundary link always lie in $G_{\omega}^{\prime}$, as the $\omega$-cover $X^{\omega}$ of the link complement $X$ 
may be constructed by splitting along Seifert surfaces [4]. In general, call an homology boundary link with group $G$ intractable if $G_{\omega}$ is perfect. For an intractable homology boundary link the longitudes are trivially in $G_{\omega}^{\prime}$. If $L$ is an intractable boundary link, then all sublinks of $L$ are intractable, and in particular all component knots have Alexander polynomial $I$. Conversely if all the component knots of a boundary link have Alexander polynomial 1 , and if all its 2-component sublinks are 2-split [16] then it is intractable. In particular the links denoted $k \cup k_{n}$ in [16], which are iterated doubles of the Whitehead link for $n \geq 1$, are intractable if $n \geq 3$. For a presentation matrix for $G_{\omega} / G_{\omega}^{\prime}=H_{1}\left(X^{\omega} ; \mathbb{Z}\right)$ as a module over $\mathbb{Z}\left[G / G_{\omega}\right]$ may be determined from the linking numbers of cycles on the Seifert surfaces as in [5]; the above assumption then implies that these linking numbers are like those of a trivial link. (However for the iterated doubles of the Whitehead link the normal closure of the longitudes in $G$ may be a proper subgroup of $G_{\omega}$.)

Kirby and Melvin showed that any knot which does not have property $R$ is (TOP)-null concordant [12], and this suggests the following complement to the above result.

THEOREM 2. If $n \geq 2$ and $L$ is a v-component $n$-link such that surgery on the longitudes of $L$ gives ${ }^{\nu}\left(S^{l} \times S^{n+1}\right)$, then $L$ is an homology boundary link and is null concordant. (Hence also any sublink of $L$ is nulz concordant.)

Proof. That $L$ is an homology boundary link is clear. Let $U(L)$ be the trace of the surgeries on $L$, so $\partial U(L)=s^{n+2} \perp \stackrel{\nu}{\nu}\left(s^{l} \times s^{n+1}\right)$. Then $D(L)=U(L) \cup\left(\stackrel{\nu}{\xi} D^{2} \times S^{n+1}\right)$ is a contractible $(n+3)$-manifold with boundary $s^{n+2}$, and so is an $(n+3)$-disc. The link $L$ clearly bounds $v$ disjoint $(n+1)$-discs in $D(L)$. //

REMARK. If $n=I$ it can be proven that $L$ bounds $v$ embedded discs in a contractible 4-manifold $W_{0}$, by imitating the first part of the theorem of Kirby and Melvin [12]. Whether the Mazur trick may be used 
to show that $W_{0}$ is $D^{4}$ may be related to the Andrews-Curtis conjecture ([11], Problem 5.7). For this comment I am indebted to Rubinstein, who has also recently proven that if 0 -framed surgery on the longitudes of the first $\rho$ components of $L$ gives $\#\left(S^{1} \times S^{2}\right)$, for each $\rho \in\{1, \ldots, \nu\}$, then $L$ is TOP null concordant [14].

In higher dimensions links which are not homology boundary links but which are sublinks of homology boundary links may be constructed as a consequence of the following theorem.

THEOREM 3. A finitely presentable group $G$ is the group of a $\mu$-component sublink of a locally flat m-link $L: U S^{m}+S^{m+2}$ (for some $\nu$ ) with group free, if and only if deficiency $G=$ weight $G=\mu$. (If $m=2$ the ambient space may be merely a homotopy 4-sphere.)

Proof. The necessity of the condition is obvious. Suppose that $G$ has a presentation $\left\langle X_{i}, 1 \leq i \leq \nu \mid r_{j}, 1 \leq j \leq \nu-\mu\right\rangle^{\phi}$ and that $s_{k}$, $1 \leq k \leq \mu$, are words in $F(\nu)$ such that the normal closure of $\left\{\phi\left(S_{i}\right) \mid 1 \leq i \leq \mu\right\}$ in $G$ is $G$. The fundamental group of \# $\left(S^{1} \times S^{m+1}\right)$ is naturally isomorphic to $F(\nu)$, and the words $r_{j}$ and $S_{k}$ may be represented by embeddings $\rho_{j}: S^{1} \rightarrow \not \nu\left(S^{1} \times S^{m+1}\right)$ and $\sigma_{k}: S^{l} \rightarrow \not \nu\left(S^{l} \times S^{m+l}\right)$ respectively. If surgery is performed on all the $\rho_{j}, 1 \leq j \leq \nu-\mu$ and $\sigma_{k}, 1 \leq k \leq \mu$, then the resulting manifold is a homotopy $(m+2)-$ sphere, and

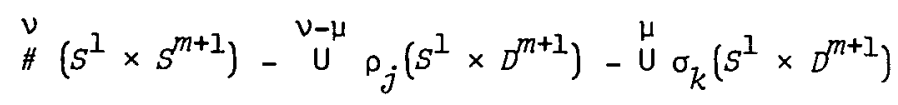

is the complement of a $v$-component m-link in this homotopy sphere with fundamental group $F(v)$ (if $m \geq 2$ ) [9]. Therefore if surgery is performed only on the $\rho_{j}, 1 \leq j \leq \nu-\mu$, the space

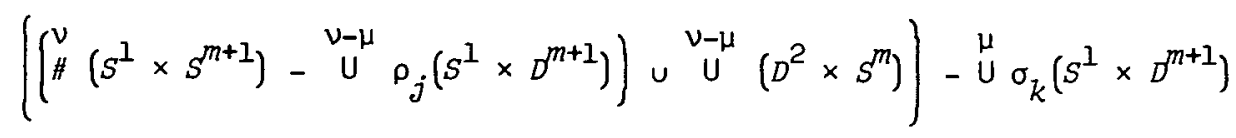


is the complement of a $\mu$-component sublink with group presented by $\left\langle x_{i}, 1 \leq i \leq \nu \mid x_{j}, 1 \leq i \leq \nu-\mu\right\rangle$, that is, with group $G$. //

If for instance $G$ has a presentation

$$
\left\langle x_{1}, x_{2}, x_{3} \mid x_{1}^{-1}\left[x_{3}^{i}, x_{1}\right]\left[x_{3}^{j}, x_{1}\right]\right\rangle
$$

where $i j \neq 0$, then according to Baumslag [1], $G$ is parafree but not free, and so cannot map onto $F(2)$. Thus the link constructed as above from this presentation is not an homology boundary link, although it is a sublink of a 3-component homology boundary link.

An imnediate consequence of Theorems 1 and 3 is that if $n>1$ the group of a $\mu$-component ribbon $n$-link has a presentation of deficiency $\mu$. Therefore for instance Fox's 2-knot with nonprincipal Alexander ideal [2] is slice [9] but not ribbon. (This was shown earlier by $\mathrm{Hit+}[8]$ and Yanagawa [19].)

It is not hard to see that a group $G$ is the group of a $\mu$-component ribbon $n$-link for $n \geq 2$ if and only if $G$ has a Wirtinger presentation of deficiency $\mu$ and $G / G^{\prime}=\mathbb{Z}^{\mu}$. (This was proven for $n=2, \mu=1$ by Yajima [18].) The argument is similar to that of the related result characterizing certain quotients of the groups of ribbon 1-links given in [6]: the generators correspond to meridional loops transverse to the components of the complements of the throughcuts, and there is one relation for each throughcut.

If $L$ is a $\nu$-component sublink of an homology boundary link (in any dimension) then the inclusion of the meridians into the link group $G$ induces isomorphisms on all the nilpotent quotients $F(\nu) / F(\nu)_{n} \rightarrow G / G_{n}$ [17]. It would be interesting to know whether this condition were sufficient for $L$ to be a sublink of an homology boundary link, in particular whether every high dimensional link be a sublink of an homology boundary link. 


\section{References}

[1] Gilbert Baumslag, "Groups with the same lower central series as a relatively free group. II. Properties", Trans. Amer. Math. Soc. 142 (1969), 507-538.

[2] R.H. Fox, "A quick trip through knot theory", Topology of 3-manifolds and related topics, 120-167 (Proc. University of Georgia Institute, 1961. Prentice-Hall, Englewood Cliffs, New Jersey, 1962).

[3] R.H. Fox, "Some problems in knot theory", Topology of 3-manifolds and related topics, 168-176 (Proc. University of Georgia Institute, 1961. Prentice-Hall, Englewood Cliffs, New Jersey, 1962).

[4] M.A. Gutiérrez, "Boundary links and an unlinking theorem", Trans. Amer. Math. Soc. 171 (1972), 491-499.

[5] M.A. Gutiérrez, "Polynomial invariants of boundary links", Rev. Colombiana Mat. 8 (1974), 97-109.

[6] Jonathan A. Hillman, "A non-homology boundary link with Alexander polynomial zero", Bull. Austral. Math. Soc. 16 (1977), 229-236.

[7] Jonathan A. Hillman, "Alexander ideals and Chen groups", Bulz. London Math. Soc. 10 (1978), 105-110.

[8] L.R. Hitt, "Examples of higher-dimensional slice knots which are not ribbon knots", preprint.

[9] Michael A. Kervaire, "Les nœuds de dimensions supérieures", Buzz. Soc. Math. France 93 (1965), 225-271.

[10] Robion Kirby, "A calculus for framed links in $S^{3}$ ", Invent. Math. 45 (1978), 35-56.

[11] Rob. Kirby, "Problems in low. dimensional manifold theory", Algebraic and geometric topology, Part 2, 273-312 (Proc. Symposia in Pure Mathematics, 32. American Mathematical Society, Providence, Rhode Island, 1978). 
[12] Robion Kirby and Paul Melvin, "Slice knots and property $\mathrm{R}$ ", Invent. Math. 45 (1978), 57-59.

[13] Valentin Poenaru, "A note on the generators for the fundamental group of the complement of a submanifold of codimension 2 ", Topology 10 (1971), 47-52.

[14] J.H. Rubinstein, "Dehn's lemma and handle decomposition of some 4-manifolds" (Mathematics Research Report, 1-1979. University of Melbourne, Parkville, Victoria, 1979).

[15] N. Smythe, "Boundary links", Topozogy Seminar, Wisconsin, 1965, 69-72 (Annals of Mathematics Studies, 60. Princeton University Press, Princeton, New Jersey, 1966).

[16] N. Smythe, "n-linking and n-splitting", Amer. J. Math. 92 (1970), $272-282$.

[17] John Stallings, "Homology and central series of groups", J. Azgebra 2 (1965), 170-181.

[18] Takeshi Yajima, "On a characterization of knot groups of some spheres in $R_{4}$ ", Osaka J. Math. 6 (1969), 435-446.

[19] Takaaki Yanagawa, "On ribbon 2-knots. The 3-manifold bounded by the 2-knots", Osaka J. Math. 6 (1969), 447-464.

Department of Pure Mathematics, School of General Studies, Australian National University, Canberra, ACT 2600, Australia.

Present Address:

Department of Mathematics, University of Durham, Durham, England. 